ÉESTI NSV TEADUSTE AKADEEEMIA TOIMÉTISED. 29. KOID̂Ë FOOSIKA * MATEMAATIKA. 1980, NR. 1

ИЗВЕСТИЯ АКАДЕМИИ НАУК ЭСТОНСКОП ССР. ТОМ 29 ФИЗИКА * МАТЕМАТИКА, 1980, № 1

П. КАРД

\title{
ОБ ОДНОМЕРНЫХ ВОЛНОВЫХ УРАВНЕНИЯХ, РЕШАЕМЫХ В БЕССЕЛЕВЫХ ФУНКЦИЯХ
}

\section{Введение}

Распространение плоской монохроматической электромагнитной волны в неоднородной слоистой среде описывается, как известно, при нормальном падении на среду одномерным волновым уравнением вида

$$
d^{2} U / d z^{2}+k^{2} n^{2}(z) U=0,
$$

где $U$ - полевая функция, $z$ - координата, отсчитываемая в неизменном направлении градиента показателя преломления $n(z), k-$ волновое число. Дисперсия не учитывается, т. е. $n$ считается не зависящим от $k$.

Известен ряд форм зависимости $n(z)$, при которых уравнение (1) решается в хорошо изученных функциях. К числу таких функций относятся, в частности, функции Бесселя. В настоящей статье мы, воспользовавшись известным преобразованием, связывающим решения двух линейных дифференциальных уравнений второго порядка (см. [ $\left.{ }^{1}\right]$, c. 151), рассмотрим обширный класс близких к волновому уравнению (1) уравнений, решения которых выражаются в виде

$$
U(z)=F(z) Z_{v}(G(z)),
$$

где $Z_{v}-$ функция Бесселя $v$-го порядка, $G(z)$ - произвольная функция, а $F(z)$ - связанная с нею другая функция. Именно, потребуем, чтобы функция $U(z)$ удовлетворяла уравнению вида

$$
d^{2} U / d z^{2}+g^{2}(z) U=0
$$

и будем искать в этом классе уравнений такие, где

$$
g(z)=k n(z)
$$

\section{Основные исходные формулы}

Подставляя функцию $U(z)$ вида $(2)$ в уравнение (3), находим

$$
Z_{v}^{\prime \prime}+\left(G^{\prime \prime} / G^{\prime 2}+2 F^{\prime} / F G^{\prime}\right) Z^{\prime}{ }_{v}+\left(F^{\prime \prime} / F G^{\prime 2}+g^{2} / G^{\prime 2}\right) Z_{v}=0
$$

где штрих означает у $Z_{v}$ производную по $G$, а у $F$ и $G$ по $z$. Это уравнение должно быть уравнением Бесселя. Следовательно,

$$
G^{\prime \prime} / G^{\prime 2}+2 F^{\prime} / F G^{\prime}=G^{-1}
$$

H

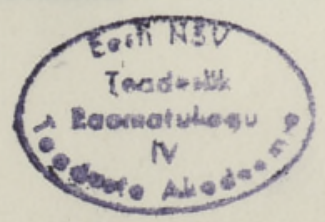




$$
F^{\prime \prime} \mid F G^{\prime 2}+g^{2} / G^{\prime 2}=1-v^{2} / G^{2} .
$$

Эти уравнения определяют общий вид функции $g(z)$, необходимый и достаточный для того, чтобы уравнение (3) имело решение вида (2). Интегрирование уравнения (6) дает

$$
F=\left(G / G^{\prime}\right)^{1 / 2} \text {. }
$$

Исключая $F$ из уравнений $(6)$ и $(7)$, находим

$$
g^{2}=G^{\prime 2}+G^{\prime \prime \prime} / 2 G^{\prime}-3 G^{\prime \prime 2} / 4 G^{\prime 2}-\left(v^{2}-1 / 4\right) G^{\prime 2} / G^{2} .
$$

Полученная формула определяет для каждой произвольной функции $G$ функцию $g^{2}$ в уравнении (3), а решение этого уравнения выражается, согласно формулам (2) и (6), в виде

$$
U=\left(G / G^{\prime}\right)^{1 / 2} Z_{v}(G) \text {. }
$$

Волновым уравнением является, однако, уравнение вида (3) не в любом случае, а только при условии, что $g(z)$ содержит постоянный фактор, отождествляемый с волновым числом (см. формулу (4)). Отсюда возникает проблема надлежащего выбора функции $G(z)$. Не претендуя на исчерпывающее решение этой проблемы, приведем несколько примеров, причем, наряду с уже известными формами показателя преломления будут получены новые формы, которые до сих пор в литературе, по-видимому, не рассматривались.

\section{Нахождение волновых уравнений}

Начнем с простейшего варианта. Приравняем нулю последние три члена формулы (9):

$$
G^{\prime \prime \prime} / 2 G^{\prime}-3 G^{\prime \prime 2} / 4 G^{\prime 2}-\left(v^{2}-1 / 4\right) G^{\prime 2} / G^{2}=0,
$$

так что

$$
g^{2}=G^{\prime 2}
$$

Дифференциальное уравнение (11) легко решается элементарными методами, причем, в силу его однородности, $G$ определяется из него с точностью до произвольного постоянного множителя. Согласно формуле (12), тот же множитель войдет и в функцию $g^{2}$, что и требуется.

Не останавливаясь на деталях, приведем результаты. Следует различать случаи $v \neq 0$ и $v=0$. Если $v \neq 0$, то

$$
G(z)=k h(A+B h / z)^{m},
$$

где

$$
m=(2 v)^{-1}
$$

а если $v=0$, то

$$
G(z)=a k h \exp (b h / z) .
$$

Здесь $k h A^{m}$ и $B h / A$ или $a k h$ и $b h-$ постоянные интегрирования. Третья постоянная $z_{0}$ принята равной нулю (иначе вместо $z$ следовало бы писать $z-z_{0}$ ) без ограничения общности, так как начало отсчета координаты произвольно. Постоянные $A, B, a, b$ безразмерны, $h$ имеет размерность длины и означает в конкретных расчетах обычно толщину слоя, $k$ - волновое число. Ниже во всех примерах $h$ и $k$ будут иметь тот же смысл, а все остальные постоянные будут безразмерны. 
Теперь, согласно формулам (3) и (12), находим следующие волновые уравнения:

$$
d^{2} U / d z^{2}+k^{2} h^{4} m^{2} B^{2} z^{-4}(A+B h / z)^{2 m-2} U=0
$$

и

$$
d^{2} U / d z^{2}+k^{2} h^{4} a^{2} b^{2} z^{-4} \exp (2 b h / z) U=0 .
$$

Решения их соответственно таковы (см. формулу (10)):

$$
U=z(A+B h / z)^{1 / 2} Z_{1 / 2 m}\left(k h(A+B h / z)^{m}\right)
$$

и

$$
U=z Z_{0}(a k h \exp (b h / z))
$$

Полученный результат можно существенно обобщить. Сохранив выражения (13) и (15) для $G(z)$, откажемся от значений $v=(2 m)^{-1}$ и $v=0$. Тогда по формуле (9) найдем

$g^{2}(z)=B^{2} h^{2} z^{-4}\left[m^{2} k^{2} h^{2}(A+B h / z)^{2 m-2}-\left(m^{2} v^{2}-1 / 4\right)(A+B h / z)^{-2}\right]$

в первом и

$$
g^{2}(z)=b^{2} h^{2} z^{-4}\left[a^{2} k^{2} h^{2} \exp (2 b h / z)-v^{2}\right]
$$

во втором случае. Затем, чтобы вторые члены этих выражений содержали множитель $k^{2}$, введем новые постоянные $S$ и $s$, положив

$$
m^{2} v^{2}-1 / 4=k^{2} h^{2} S
$$

в первом и

$$
v=k h s
$$

во втором случае. Тогда получим следующие волновые уравнения с их решениями:

$$
\begin{gathered}
d^{2} U / d z^{2}+B^{2} k^{2} h^{4} z^{-4}(A+B h / z)^{-2}\left[m^{2}(A+B h / z)^{2 m}-S\right] U=0, \\
U=z(A+B h / z)^{1 / 2} Z_{v}\left(k h(A+B h / z)^{m}\right),
\end{gathered}
$$

где

$$
v=(2 m)^{-1}\left(1+4 k^{2} h^{2} S\right)^{1 / 2}
$$

и

$$
\begin{gathered}
d^{2} U / d z^{2}+b^{2} k^{2} h^{4} z^{-4}\left[a^{2} \exp (2 b h / z)-s^{2}\right] U=0, \\
U=z Z_{k h s}(a k h \exp (b h / z)) .
\end{gathered}
$$

Прежние результаты (формулы (16)-(19)) получаются отсюда как частные случаи при $S=0$ или $S=0$, соответственно.

Перейдем к дальнейшему обобщению. Примем в формуле (9)

$$
G(z)=p k h\left(\frac{a z / h+b}{c z / h+d}\right)^{m}
$$

где

$$
a d-b c=1 .
$$

Вычисляя по формуле (9), находим 


$$
\begin{gathered}
g^{2}(z)=k^{2} p^{2} m^{2}(a z / h+b)^{2 m-2}(c z / h+d)^{-2 m-2}- \\
-h^{-2}\left(m^{2} v^{2}-1 / 4\right)(a z / h+b)^{-2}(c z / h+d)^{-2} .
\end{gathered}
$$

Второй член должен, как и первый, содержать множитель $k^{2}$. Поэтому положим

$$
m^{2} v^{2}-1 / 4=k^{2} h^{2} S .
$$

В результате получаем волновое уравнение

$$
\begin{gathered}
d^{2} U / d z^{2}+k^{2}(a z / h+b)^{-2}(c z / h+d)^{-2} \times \\
\times\left[p^{2} m^{2}(a z / h+b)^{2 m}(c z / h+d)^{-2 m}-S\right] U=0,
\end{gathered}
$$

решение которого имеет вид

$$
U=(a z / h+b)^{1 / 2}(G z / h+d)^{1 / 2} Z_{v}\left(p k h\left(\frac{a z / h+b}{c z / h+d}\right)^{m}\right),
$$

где

$$
v=(2 m)^{-1}\left(1+4 k^{2} h^{2} S\right)^{1 / 2} .
$$

Это уравнение является, очевидно, обобщением уравнения (24).

Аналогичному обобщению поддается и уравнение (27). Для этого следует в формуле (9) положить

$$
G(z)=k h \exp \left(\frac{p(a z / h+b)}{c z / h+d}\right), \quad a d-b c=1 .
$$

Вычисляя и беря

$$
v=k h s \text {, }
$$

находим волновое уравнение

$$
d^{2} U / d z^{2}+k^{2} p^{2}(c z / h+d) \rightarrow\left\{\exp \left(\frac{2 p(a z / h+b)}{c z / h+d}\right)-s^{2}\right\} U=0
$$

и его решение

$$
U=(c z / h+d) Z_{k h s}\left(k h \exp \left(\frac{p(a z / h+b)}{c z / h+d}\right)\right) .
$$

Напоследок приведем еще два примера. Пусть, во-первых,

$$
G(z)=\left\{A+B \arctan \left(\frac{a z / h+b}{c z / h+d}\right)\right\}^{p}, \quad a d-b c=1 .
$$

Подставляя эту функцию в формулу (9), находим

$$
\begin{array}{r}
g^{2}(z)=h^{-2}\left[\left(a^{2}+c^{2}\right) z^{2} / h^{2}+2(a b+c d) z / h+\left(b^{2}+d^{2}\right)\right]^{-2} \times \\
\times\left\{B^{2} p^{2}\left[A+B \arctan \left(\frac{a z / h+b}{c z / h+d}\right)\right]^{2 p-2}-\right. \\
\left.-B^{2}\left(v^{2} p^{2}-1 / 4\right)\left[A+B \arctan \left(\frac{a z / h+b}{c z / h+d}\right)\right]^{-2}-1\right\} .
\end{array}
$$


Чтобы это выражение дало волновое уравнение, нужно выделить множитель $k^{2}$. Для этого положим

$$
\begin{gathered}
p=3 / 2, \\
v=1 / 3, \\
B=(k h / p)^{2 / 3} C^{1 / 3}=(2 k h / 3)^{2 / 3} C^{1 / 3}
\end{gathered}
$$

Тогда формула (41) принимает желаемый вид

$$
\begin{gathered}
g^{2}(z)=k^{2}\left[\left(a^{2}+c^{2}\right) z^{2} / h^{2}+2(a b+c d) z / h+\left(b^{2}+d^{2}\right)\right]^{-2} \times \\
\times C \arctan \left(\frac{a z / h+b}{c z / h+d}\right),
\end{gathered}
$$

а для $G(z)$ из формулы (40) находим выражение

$$
G(z)=\left(2 / 3 k^{2} h^{2} C\right)\left\{1+C k^{2} h^{2} \arctan \left(\frac{a z / h+b}{c z / h+d}\right)\right\}^{3 / 2}
$$

Волновое уравнение получается в виде

$$
\begin{gathered}
d^{2} U / d z^{2}+k^{2}\left[\left(a^{2}+c^{2}\right) z^{2} / h^{2}+2(a b+c d) z / h+\left(b^{2}+d^{2}\right)\right]^{-2} \times \\
\times C \arctan \left(\frac{a z / h+b}{c z / h+d}\right) U=0
\end{gathered}
$$

и имеет решение

$$
\begin{gathered}
U=\left[\left(a^{2}+c^{2}\right) z^{2} / h^{2}+2(a b+c d) z / h+\left(b^{2}+d^{2}\right)\right]^{1 / 2} \times \\
\times\left\{1+C k^{2} h^{2} \arctan \left(\frac{a z / h+b}{c z / h+d}\right)\right\}^{1 / 2} \times \\
\times Z_{1 / 2}\left(\left(2 / 3 k^{2} h^{2} C\right)\left\{1+C k^{2} h^{2} \arctan \left(\frac{a z / h+b}{c z / h+d}\right)\right\}^{3 / 2}\right) .
\end{gathered}
$$

Наш последний пример аналогичен предыдущему. Возьмем

$$
G(z)=\left(A+B \ln \left(\frac{a z / h+b}{c z / h+d}\right)\right)^{p}, \quad a d-b c=1 .
$$

Формула (9) дает

$$
\begin{aligned}
g^{2}(z)= & h^{-2}(a z / h+b)^{-2}(c z / h+d)^{-2}\left\{B^{2} p^{2}\left[A+B \ln \left(\frac{a z / h+b}{c z / h+d}\right)\right]^{2 p-2}-\right. \\
& \left.-B^{2}\left(v^{2} p^{2}-1 / 4\right)\left[A+B \ln \left(\frac{a z / h+b}{c z / h+d}\right)\right]^{-2}+1 / 4\right\}
\end{aligned}
$$

Здесь положим, как и в формуле (40), $p=3 / 2, v=1 / 3, B=$ $=(2 \mathrm{kh} / 3)^{2 / 3} C^{1 / 3}(\mathrm{~cm}$. формулы $(42)-(44))$, но формулу $(45)$ несколько изменим:

$$
A=-(2 B p)^{-2}=-1 / 9 B^{2}
$$


Тогда

$$
g^{2}(z)=k^{2}(a z / h+b)^{-2}(c z / h+d)^{-2} C \ln \left(\frac{a z / h+b}{c z / h+d}\right)
$$

и

$$
G(z)=\left(12 k^{2} h^{2} C\right)^{-1}\left\{-1+4 k^{2} h^{2} C \ln \left(\frac{a z / h+b}{c z / h+d}\right)\right\}^{3 / 2}
$$

Итак, мы нашли волновое уравнение

$$
d^{2} U / d z^{2}+k^{2}(a z / h+b)^{-2}(c z / h+d)^{-2} C \ln \left(\frac{a z / h+b}{c z / h+d}\right) U=0
$$

с его решением

$$
\begin{aligned}
U= & (a z / h+b)^{1 / 2}(c z / h+d)^{1 / 2}\left\{-1+4 k^{2} h^{2} C \ln \left(\frac{a z / h+b}{c z / h+d}\right)\right\}^{1 / 2} \times \\
& \times Z_{1 / s}\left(\left(12 k^{2} h^{2} C\right)^{-1}\left\{-1+4 k^{2} h^{2} C \ln \left(\frac{a z / h+b}{c z / h+d}\right)\right\}^{3 / 2}\right) .
\end{aligned}
$$

В заключение приведем сводку четырех рассмотренных здесь наиболее общих форм зависимости показателя преломления от координаты, при которых решение волнового уравнения выражается через функции Бесселя:

$$
\begin{gathered}
n(z)=(a z / h+b)^{-1}(c z / h+d)^{-1}\left\{p^{2} m^{2}(a z / h+b)^{2 m}(c z / h+d)^{-2 m}-S\right\}^{1 / 2} \\
n(z)=p(c z / h+d)^{-2}\left\{\exp \left(\frac{2 p(a z / h+b)}{c z / h+d}\right)-s^{2}\right\}^{1 / 2} \\
n(z)=\left[\left(a^{2}+c^{2}\right) z^{2} / h^{2}+2(a b+c d) z / h+\left(b^{2}+d^{2}\right)\right]^{-1} \times \\
\times\left\{C \arctan \left(\frac{a z / h+b}{c z / h+d}\right)\right\}^{1 / 2}
\end{gathered}
$$

и

$$
n(z)=(a z / h+b)^{-1}(c z / h+d)^{-1}\left\{C \ln \left(\frac{a z / h+b}{c z / h+d}\right)\right\}^{t / 2} .
$$

Эти формы входят в уравнения (33), (38), (48) и (55). Уравнения (16), $(17),(24)$ и $(27)$ являются частными видами названных общих уравнений.

\section{Л И Т Е Р А Т Р А}

1. К а м ке Э., Справочник по обыкновенным дифференциальным уравнениям, изд. 2-е, М., ГИФМЛ, 1961. 


\section{BESSELI FUNKTSIOONIDE KAUDU LAHENDUVATEST OHEMOOTMELISTEST LAINEVORRANDITEST}

Kui diferentsiaalvõrrandis (3) oleval funktsioonil $g^{2}(z)$ on kuju (9), kus $G(z)$ on meelevaldne funktsioon, siis avaldub võrrandi lahend valemiga (10), kus $Z_{v}$ on $v$-ndat järku Besseli funktsioon. Funktsiooni $G(z)$ sobiva valiku korral $g(z)=k n(z)$, mispuhul võrrand (3) saab ühemõõtmeliseks lainevôrrandiks (1), mis kirjeldab monokromaatilise (lainearvuga $k$ ) valguse levimist mittehomogeenses kihilises keskkonnas, mille murdumisnäitaja on $n(\boldsymbol{z})$. Artiklis on leitud neli sellekohast üldjuhtu. Murdumisnäitaja avaldub neil juhtudel valemitega (57)-(60), kus $a, b, c, d, m, p, s, S$ ja $C$ on dimensioonitud konstandid (kusjuures igal pool $a d-b c=1$ ) ja $h$ on pikkuse dimensiooniga suurus (tavaliselt kihi paksus). Lainevõrrandid on vastavalt valemites (33), (38), (48) ja (55) ning funktsioon $G(z)$ valemites (29), (36), (47) ja (54), kuna Besseli funktsiooni järk on esimesel juhul $(2 m)^{-1}\left(1+4 k^{2} h^{2} S\right)^{1 / 2}$, teisel juhul khs ja kahel viimasel juhul $1 / 3$.

\section{P. KARD}

\section{ON THE ONE-DIMENSIONAL WAVE EQUATIONS HAVING SOLUTIONS IN TERMS OF BESSEL FUNCTIONS}

If the function $g^{2}(z)$ entering the differential equation (3) is of the form (9), where $G(z)$ is an arbitrary function, then the solution of this equation is done by the formula (10), where $Z_{v}$ is Bessel function of the $v$-th order. Suitable choice of the function $G(z)$ renders the function $g(z)$ equal to $k n(z)$, whereby the equation (3) becomes identical with the one-dimensional wave equation (1) describing the propagation of monochromatic light of the wave number $k$ through inhomogeneous layered medium with the refractive index $n(z)$. In this article four general forms of such equations are found. The refractive indices for these cases are put down in the formulae $(57)-(60)$, where $a, b, c, d, m, p, s, S$ and $C$ are dimensionless constants (with $a d-b c=1$ everywhere), whereas $h$ has the dimension of length and means usually the thickness of the layer. The wave equations with these refractive indices are given in the formulae (33), (38), (48), and (55), and the function $G(z)$ in the formulae (29), (36), (47), and (54). The order of Bessel function is in the first case $(2 m)^{-1}\left(1+4 k^{2} h^{2} S\right)^{1 / 2}$, in the second case $k h s$, and in the last two cases $1 / 3$. 